\title{
DUALITY IN THE OPTIMAL CONTROL FOR DAMPED HYPERBOLIC SYSTEMS WITH POSITIVE CONTROL
}

\author{
MI JIN LEE, JONG YEOUL PARK, and YOUNG CHEL KWON
}

\author{
Received 10 September 2002
}

\begin{abstract}
We study the duality theory for damped hyperbolic equations. These systems have positive controls and convex cost functionals. Our main results lie in the application of duality theorem, that is, $\inf J=\sup K$, on various cost functions.
\end{abstract}

2000 Mathematics Subject Classification: 49J20, 49N15, 93C20.

1. Introduction. Lions [2] introduced optimal control problems of the variety of distributed parameter systems, for example, elliptic, parabolic, and hyperbolic. Here, we see that adjoint state systems are given cost functional and distributed parameter systems. Duality theory is $\inf J=\sup K$, where $J$ satisfies systems and $K$ satisfies adjoint state systems. The duality theory for the corresponding parabolic systems has been given by Chan [1] and Tanimoto [7]. Park and Lee [5, 6] studied the duality theory for hyperbolic systems. Also, they [4] obtained same results for hyperbolic systems with damping terms. In this paper, we study the duality theory for damped hyperbolic systems with positive controls. These systems have various convex cost functionals. The main objective is to prove the duality theorem for damped hyperbolic systems with positive controls and various cost functions. The main tools are integration by parts and Green's formula.

2. Preliminaries. Let $X$ be a Hilbert space $(\cdot, \cdot)$ and let $\|\cdot\|_{X}$ denote the inner product and the induced norm on $X ; X^{\prime}$ denotes the dual space of $X$ and $\langle\cdot, \cdot\rangle_{X^{\prime}, X}$ denotes the dual pairing between $X^{\prime}$ and $X$. We introduce underlying Hilbert spaces to describe damped second-order evolution equations. Let $H$ be a real pivot Hilbert space; its norm $\|\cdot\|_{H}$ is simply denoted by $|\cdot|_{H}$. For $i=1,2$, let $V_{i}$ be a real separable Hilbert space. Assume that each pair $\left(V_{i}, H\right)$ is a Gelfand triple space with a notation $V_{i} \hookrightarrow H \equiv H^{\prime} \hookrightarrow V_{i}^{\prime}$. We suppose that $V_{1}$ is continuously embedded in $V_{2}$. Then we see that $V_{1} \hookrightarrow V_{2} \hookrightarrow H \equiv H^{\prime} \hookrightarrow$ $V_{2}^{\prime} \hookrightarrow V^{\prime}$ and the equalities $\langle\phi, \psi\rangle_{V_{1}^{\prime}, V_{1}}=\langle\phi, \psi\rangle_{V_{2}^{\prime}, V_{2}}$ for $\phi \in V_{2}^{\prime}, \psi \in V$, and $\langle\phi, \psi\rangle_{V_{1}^{\prime}, V_{1}}=(\phi, \psi)_{H}$ for $\phi \in H, \psi \in V$ hold. Let $T$ be a positive number. We define a function space $W(0, T)$ by

$$
W(0, T)=\left\{y \mid y \in L^{2}\left(0, T ; V_{1}\right), y^{\prime} \in L^{2}\left(0, T ; V_{2}\right), y^{\prime \prime} \in L^{2}\left(0, T ; V_{1}^{\prime}\right)\right\}
$$


with an inner product

$$
\left(y_{1}, y_{2}\right)_{W(0, T)}=\int_{0}^{T}\left\{\left(y_{1}(t), y_{2}(t)\right)_{V_{1}}+\left(y_{1}^{\prime}(t), y_{2}^{\prime}(t)\right)_{V_{2}}+\left(y_{1}^{\prime \prime}(t), y_{2}^{\prime \prime}(t)\right)_{V_{1}^{\prime}}\right\} d t
$$

This becomes a Hilbert space with norm

$$
\|y\|_{W(0, T)}=\left(\|y\|_{L^{2}\left(0, T ; V_{1}\right)}^{2}+\left\|y^{\prime}\right\|_{L^{2}\left(0, T ; V_{2}\right)}^{2}+\left\|y^{\prime \prime}\right\|_{L^{2}\left(0, T ; V_{1}^{\prime}\right)}^{2}\right)^{1 / 2},
$$

where ${ }^{\prime}=d / d t$ and ${ }^{\prime \prime}=d^{2} t / d t^{2}$.

From now on, we set $V=V_{2}=H_{0}^{1}(\Omega)$ and $H=L^{2}(\Omega)$, where $\Omega$ is a bounded open set in $\mathbb{R}^{n}$ with smooth boundary $\Gamma$, and let $Q=\Omega \times(0, T)$. We will give an exact description of damped second-order evolution equations. We consider the bilinear forms defined by

$$
\begin{aligned}
a_{1}(t ; \phi, \psi)= & \sum_{i, j=1}^{n} \int_{\Omega} a_{i j}(t, x) \frac{\partial \phi(x)}{\partial x_{i}} \frac{\partial \psi(x)}{\partial x_{j}} d x \\
& +\int_{\Omega} a_{0}(t, x) \phi(x) \psi(x) d x \quad \forall \phi, \psi \in V, \\
a_{2}(t ; \phi, \psi)= & \sum_{i, j=1}^{n} \int_{\Omega} b_{i j}(t, x) \frac{\partial \phi(x)}{\partial x_{i}} \frac{\partial \psi(x)}{\partial x_{j}} d x \\
& +\int_{\Omega} b_{0}(t, x) \phi(x) \psi(x) d x \quad \forall \phi, \psi \in V,
\end{aligned}
$$

where $a_{i j}, b_{i j}, a_{0}$, and $b_{0}$ are the functions satisfying the following properties:

(i) $a_{i j}=a_{j i}$ and $b_{i j}=b_{j i}$,

(ii) $a_{i j}, b_{i j}, a_{0}, b_{0} \in C^{1}\left([0, T] ; L^{\infty}(\Omega)\right)$,

(iii) $\sum_{i, j=1}^{n} a_{i, j}(t, x) \xi_{i} \xi_{j} \geq c_{1}\left(\xi_{1}^{2}+\cdots+\xi_{n}^{2}\right), c_{1}>0, \xi_{i} \in \mathbb{R}$,

(iv) $\sum_{i, j=1}^{n} b_{i, j}(t, x) \xi_{i} \xi_{j} \geq c_{2}\left(\xi_{1}^{2}+\cdots+\xi_{n}^{2}\right), c_{2}>0, \xi_{i} \in \mathbb{R}$.

Using the above properties, we can show the coercivity condition of $a_{1}$ and $a_{2}$. Indeed, by (i) and (ii), there exists $K>0$ such that $\left|a_{0}(t, x)\right| \leq K$ a.e., $x \in \Omega$, and for all $t \in[0, T]$. The coercivity condition of $a_{1}$ follows from

$$
\begin{aligned}
a_{1}(t ; \phi, \psi) & \geq c_{1} \sum_{i=1}^{n} \int_{\Omega}\left|\frac{\partial \phi(x)}{\partial x_{i}}\right|^{2} d x-K \int_{\Omega}|\phi(x)|^{2} d x \\
& \geq c_{1}\|\phi\|_{H_{0}^{1}(\Omega)}^{2}-K|\phi|_{L^{2}(\Omega)}^{2} .
\end{aligned}
$$

Similarly, we can show the coercivity condition of $a_{2}$. Then we can define the operator $A_{i}(t) \in \mathscr{L}\left(V_{i}, V_{i}^{\prime}\right)$ for $t \in[0, T]$ deduced by the relation

$$
a_{i}(t ; \phi, \psi)=\left\langle A_{i}(t) \phi, \psi\right\rangle_{V_{i}^{\prime}, V_{i}} \quad \forall \phi, \psi \in V_{i}, i=1,2
$$


Let $y_{0} \in H_{0}^{1}(\Omega)$ and $y_{1} \in L^{2}(\Omega)$. Then by Nakagiri and Ha [3], there exists a solution $y \in W(0, T)$ of

$$
\begin{gathered}
\frac{\partial^{2} y(u ; t)}{\partial t^{2}}+A_{2}(t) \frac{\partial y(u ; t)}{\partial t}+A_{1}(t) y(u ; t)=u \quad \text { in }(0, T) \\
y(u ; 0)=y_{0}, \quad y^{\prime}(u ; 0)=y_{1} \quad \text { in } \Omega, \quad y=0 \quad \text { on } \Sigma .
\end{gathered}
$$

3. Duality. We choose a control variable space $u=L^{2}(Q)$. Let $u \in U_{a d}=$ $\{u \mid u \geq 0$ a.e. in $Q\} \subset \mathcal{U}$ and $y$ satisfying (2.7). we consider a cost functional given by

$$
J(y, u)=\frac{1}{2} \int_{0}^{T}\left|y(u)-z_{d}\right|^{2} d t+\frac{1}{2} \int_{0}^{T}(N u, u) d t
$$

For our purpose, we consider the following systems:

$$
\begin{aligned}
& y^{\prime \prime}(u ; t)+A_{2}(t) y^{\prime}(u ; t)+A_{1}(t) y(u ; t)=u \quad \text { in } Q, \\
& y(u ; 0)=y_{0}, \quad y^{\prime}(u ; 0)=y_{1} \quad \text { in } \Omega, \\
& u \geq 0 \quad \text { a.e. in } Q, \quad y(0) \geq 0, \quad y^{\prime}(0) \geq 0, \quad y=0 \quad \text { on } \Sigma, \\
& p^{\prime \prime}(u ; t)-A_{2}(t) p^{\prime}(u ; t)+\left(A_{1}(t)-A_{2}^{\prime}(t)\right) p(u ; t)=y-z_{d} \quad \text { in } Q, \\
& p=0 \quad \text { on } \Sigma, \quad p(u ; T)=0, \quad p^{\prime}(u ; T)=0 \\
& p+N u \geq 0 \quad \text { in } Q, \quad p(0) \geq 0, \quad p^{\prime}(0) \geq 0, \\
& u(p+N u)=0, \\
& p(0) y(0)=0 \quad \text { in } \Omega, \quad y^{\prime}(0) p(0)=0, \quad y(0) p^{\prime}(0)=0 .
\end{aligned}
$$

THEOREM 3.1. Let $J=(1 / 2) \int_{0}^{T}\left|y-z_{d}\right|^{2} d t+(1 / 2) \int_{0}^{T}(N u, u) d t$ and $K=$ -(1/2) $\int_{0}^{T}|y|^{2} d t+(1 / 2) \int_{0}^{T}\left|z_{d}\right|^{2} d t-(1 / 2) \int_{0}^{T}(N u, u) d t$. Assume that $y_{0}, u_{0}$, and $p_{0}$ satisfy (3.2), (3.3), and (3.4), respectively, $y$ and $u$ in $J$ satisfy (3.2), and $y$ and $u$ in $K$ satisfy (3.3). Then

$$
\inf _{(3.2)} J=J\left(y_{0}, u_{0}\right)=K\left(y_{0}, u_{0}\right)=\sup _{(3.3)} K
$$

Proof. (i) We begin by showing that $J=K$ at $\left(y_{0}, u_{0}, p_{0}\right)$.

$$
\begin{aligned}
J\left(y_{0}, u_{0}\right) & =J\left(y_{0}, u_{0}\right)-\int_{0}^{T}\left(u_{0}, p_{0}\right) d t-\int_{0}^{T}\left(u_{0}, N u_{0}\right) d t \\
& =J\left(y_{0}, u_{0}\right)-\int_{0}^{T}\left(y_{0}^{\prime \prime}+A_{2}(t) y_{0}^{\prime}+A_{1}(t) y_{0}, p_{0}\right) d t-\int_{0}^{T}\left(u_{0}, N u_{0}\right) d t
\end{aligned}
$$




$$
\begin{aligned}
= & J\left(y_{0}, u_{0}\right)-\int_{0}^{T}\left(y_{0}, p_{0}^{\prime \prime}-A_{2}^{\prime}(t) p_{0}-A_{2}(t) p_{0}^{\prime}+A_{1}(t) p_{0}\right) d t \\
& -\int_{0}^{T}\left(u_{0}, N u_{0}\right) d t \\
= & J\left(y_{0}, u_{0}\right)-\int_{0}^{T}\left(y_{0}, y_{0}-z_{d}\right) d t-\int_{0}^{T}\left(u_{0}, N u_{0}\right) d t .
\end{aligned}
$$

(ii) To show $\inf _{(3.2)} J=J\left(y_{0}, u_{0}\right)$, we must check that $J(y, u) \geq J\left(y_{0}, u_{0}\right)$ under (3.2) for $(y, u, p)$ and under (3.2), (3.3), and (3.4) for $\left(y_{0}, u_{0}, p_{0}\right)$. Now, we have

$$
\begin{aligned}
J(y, u)-y\left(y_{0}, u_{0}\right) \geq & \int_{0}^{T}\left(y_{0}-z_{d}, y-y_{0}\right) d t+\int_{0}^{T}\left(N u_{0}, u-u_{0}\right) d t \\
= & \int_{0}^{T}\left(p_{0}^{\prime \prime}-A_{2}(t) p_{0}^{\prime}+\left(A_{1}(t)-A_{2}^{\prime}(t)\right) p_{0}, y-y_{0}\right) d t \\
& +\int_{0}^{T}\left(N u_{0}, u-u_{0}\right) d t \\
= & \int_{0}^{T}\left(p_{0}, y^{\prime \prime}+A_{2}(t) y^{\prime}+A_{1}(t) y\right) d t \\
& -\int_{0}^{T}\left(p_{0}, y_{0}^{\prime \prime}+A_{2}(t) y_{0}^{\prime}+A_{1}(t) y_{0}\right) d t \\
& +\int_{0}^{T}\left(N u_{0}, u-u_{0}\right) d t \\
= & \int_{0}^{T}\left(p_{0}, u-u_{0}\right) d t+\int_{0}^{T}\left(N u_{0}, u-u_{0}\right) d t \\
\geq & 0 .
\end{aligned}
$$

(iii) We claim that $K(y, u) \leq K\left(y_{0}, u_{0}\right)$ under (3.3) for $(y, u, p)$ :

$$
\begin{aligned}
J\left(y_{0}, u_{0}\right)-J(y, u) \\
\geq \int_{0}^{T}\left(y-z_{d}, y-y_{0}\right) d t+\int_{0}^{T}\left(N u, u-u_{0}\right) d t \\
=\int_{0}^{T}\left(p^{\prime \prime}-A_{2}(t) p^{\prime}+\left(A_{1}(t)-A_{2}^{\prime}(t)\right) p, y_{0}-y\right) d t+\int_{0}^{T}\left(N u, u_{0}-u\right) d t \\
\quad+\int_{0}^{T}\left(y_{0}^{\prime \prime}+A_{2}(t) y_{0}^{\prime}+A_{1}(t) y_{0}-u_{0}, p_{0}-p\right) d t \\
=-\int_{0}^{T}\left(y-z_{d}, y\right) d t-\int_{0}^{T}(N u, u) d t+\int_{0}^{T}\left(y_{0}, y_{0}-z_{d}\right) d t \\
\quad+\int_{0}^{T}\left(N u_{0}, u_{0}\right) d t+\int_{0}^{T}\left(N u+p, u_{0}\right) d t-\int_{0}^{T}\left(u_{0}, p_{0}+N u_{0}\right) d t
\end{aligned}
$$




$$
\begin{aligned}
\geq & -\int_{0}^{T}\left(y-z_{d}, y\right) d t-\int_{0}^{T}(N u, u) d t \\
& +\int_{0}^{T}\left(y_{0}, y_{0}-z_{d}\right) d t+\int_{0}^{T}\left(N u_{0}, u_{0}\right) d t .
\end{aligned}
$$

Therefore

$$
K\left(y_{0}, u_{0}\right) \geq K(y, u)
$$

This completes the proof.

Now, we observe the terminal value of $y(u ; t)$. Since the observation $z(u)$ is given by $y(u ; T)$, the cost function is given as

$$
J(y, u)=\frac{1}{2}\left|y(T)-z_{d}\right|^{2}+\frac{1}{2} \int_{0}^{T}(N u, u) d t
$$

We introduce the following systems:

$$
\begin{aligned}
& y^{\prime \prime}(u ; t)+A_{2}(t) y^{\prime}(u ; t)+A_{1}(t) y(u ; t)=u \quad \text { in } Q, \\
& y(u ; 0)=y_{0}, \quad y^{\prime}(u ; 0)=y_{1} \quad \text { in } \Omega, \\
& u \geq 0 \quad \text { a.e. in } Q, \quad y(0) \geq 0, \quad y^{\prime}(0) \geq 0, \quad y=0 \quad \text { on } \Sigma, \\
& p^{\prime \prime}(u ; t)-A_{2}(t) p^{\prime}(u ; t)+\left(A_{1}(t)-A_{2}^{\prime}(t)\right) p(u ; t)=0 \quad \text { in } Q, \\
& p=0 \quad \text { on } \Sigma, \\
& p(u ; T)=0, \quad p^{\prime}(u ; T)=y(T)-z_{d}, \\
& -p+N u \geq 0 \quad \text { in } Q, \quad p(0) \geq 0, \quad p^{\prime}(0) \geq 0, \\
& u(-p+N u)=0, \\
& p(0) y(0)=0 \quad \text { in } \Omega, \quad y^{\prime}(0) p(0)=0, \quad y(0) p^{\prime}(0)=0 .
\end{aligned}
$$

THEOREM 3.2. Let $J=(1 / 2)\left|y(T)-z_{d}\right|^{2}+(1 / 2) \int_{0}^{T}(N u, u) d t$ and $K=$ $-(1 / 2)|y(T)|^{2}+(1 / 2)\left|z_{d}\right|-(1 / 2) \int_{0}^{T}(N u, u) d t$. Assume that $y_{0}, u_{0}$, and $p_{0}$ satisfy (3.11), (3.12), and (3.13), respectively, $y$ and $u$ in $J$ satisfy (3.11), and $y$ and $u$ in $K$ satisfy (3.12). Then

$$
\inf _{(3.11)} J=J\left(y_{0}, u_{0}\right)=K\left(y_{0}, u_{0}\right)=\sup _{(3.12)} K
$$


Proof. (i) We now prove that $J\left(y_{0}, u_{0}\right)=K\left(y_{0}, u_{0}\right)$ :

$$
\begin{aligned}
J\left(y_{0}, u_{0}\right) & =J\left(y_{0}, u_{0}\right)+\int_{0}^{T}\left(p_{0}, u_{0}\right) d t-\int_{0}^{T}\left(N u_{0}, u_{0}\right) d t \\
& =J\left(y_{0}, u_{0}\right)+\int_{0}^{T}\left(y_{0}^{\prime \prime}+A_{2}(t) y_{0}^{\prime}+A_{1}(t) y_{0}, p_{0}\right) d t-\int_{0}^{T}\left(N u_{0}, u_{0}\right) d t \\
& =\frac{1}{2}\left|y_{0}(T)-z_{d}\right|^{2}-\left(y_{0}(T)-z_{d}, y_{0}(T)\right)-\frac{1}{2} \int_{0}^{T}\left(N u_{0}, u_{0}\right) d t \\
& =-\frac{1}{2}\left|y_{0}(T)\right|^{2}+\frac{1}{2}\left|z_{d}\right|^{2}-\frac{1}{2} \int_{0}^{T}\left(N u_{0}, u_{0}\right) d t \\
& =K\left(y_{0}, u_{0}\right) .
\end{aligned}
$$

(ii) We show that $J(y, u) \geq J\left(y_{0}, u_{0}\right)$ under (3.11) for $(y, u, p)$ and under (3.11), (3.12), and (3.13) for $\left(y_{0}, u_{0}, p_{0}\right)$ :

$$
\begin{aligned}
J(y, u)-J\left(y_{0}-u_{0}\right) \geq & \left(y_{0}(T)-z_{d}, y(T)-y_{0}(T)\right)+\int_{0}^{T}\left(N u_{0}, u-u_{0}\right) d t \\
= & \left(y_{0}(T)-z_{d}, y(T)-y_{0}(T)\right)+\int_{0}^{T}\left(N u_{0}, u-u_{0}\right) d t \\
& -\int_{0}^{T}\left(p^{\prime \prime}-A_{2}(t) p^{\prime}+\left(A_{1}(t)-A_{2}^{\prime}(t)\right) p, y-y_{0}\right) d t \\
= & \left(y_{0}(T)-z_{d}, y(T)-y_{0}(T)\right) \\
& +\int_{0}^{T}\left(N u_{0}, u-u_{0}\right) d t-\left(y_{0}(T)-z_{d}, y(T)-y_{0}(T)\right) \\
& -\int_{0}^{T}\left(p_{0}, y^{\prime \prime}-y_{0}^{\prime \prime}+A_{2}(t) y^{\prime}-A_{2}(t) y_{0}^{\prime}\right. \\
= & \int_{0}^{T}\left(-p_{0}+N u_{0}, u-u_{0}\right) d t \\
\geq & 0 .
\end{aligned}
$$

(iii) We have to check that $K(y, u) \leq K\left(y_{0}, u_{0}\right)$ under (3.12) for $(y, u, p)$ :

$$
\begin{aligned}
J\left(y_{0}, u_{0}\right)-J(y, u) \geq & \left(y(T)-z_{d}, y_{0}(T)-y(T)\right)+\int_{0}^{T}\left(N u, u_{0}-u\right) d t \\
= & \left(y(T)-z_{d}, y_{0}(T)-y(T)\right)+\int_{0}^{T}\left(N u, u_{0}-u\right) d t \\
& -\int_{0}^{T}\left(y_{0}^{\prime \prime}+A_{2}(t) y_{0}^{\prime}+A_{1}(t) y_{0}-u_{0}, p_{0}-p\right) d t
\end{aligned}
$$




$$
\begin{aligned}
= & -\left(y(T)-z_{d}, y(T)\right)-\int_{0}^{T}(N u, u)+\left(y_{0}(T)-z_{d}, y_{0}(T)\right) d t \\
& +\int_{0}^{T}\left(N u_{0}, u_{0}\right) d t+\int_{0}^{T}\left(-p+N u, u_{0}\right) d t \\
& +\int_{0}^{T}\left(p_{0}-N u_{0}, u_{0}\right) d t \\
\geq & -\left(y(T)-z_{d}, y(T)\right)-\int_{0}^{T}(N u, u) d t \\
& +\left(y_{0}(T)-z_{d}, y_{0}(T)\right)+\int_{0}^{T}\left(N u_{0}, u_{0}\right) d t .
\end{aligned}
$$

This shows that

$$
K\left(y_{0}, u_{0}\right) \geq K(y, u)
$$

Therefore, Theorem 3.2 is proved.

When the observation $z(u)$ is given by $z(u)=y^{\prime}(u)$, the cost function is defined as

$$
J\left(y^{\prime}, u\right)=\frac{1}{2} \int_{0}^{T}\left|y^{\prime}(u)-z_{d}\right|^{2} d t+\frac{1}{2} \int_{0}^{T}(N u, u) d t .
$$

We will consider the following systems:

$$
\begin{aligned}
& y^{\prime \prime}(u ; t)+A_{2}(t) y^{\prime}(u ; t)+A_{1}(t) y(u ; t)=u \quad \text { in }(0, T), \\
& y(u ; 0)=y_{0}, \quad y^{\prime}(u ; 0)=y_{1} \quad \text { in } \Omega, \\
& u \geq 0 \quad \text { a.e. in } Q, \quad y(0) \geq 0, \quad y^{\prime}(0) \geq 0, \quad y=0 \quad \text { on } \Sigma, \\
& p^{\prime \prime}(u ; t)-A_{2}(t) p^{\prime}(u ; t)+A_{1}(t) p(u ; t)+\int_{t}^{T} A_{1}^{\prime}(\sigma) p(u ; \sigma) d \sigma \\
& \quad=y^{\prime}(u ; t)-z_{d} \quad \text { in } Q, \\
& p=0 \quad \text { on } \Sigma, \quad p(u ; T)=0, \quad p^{\prime}(u ; T)=0, \\
& -p^{\prime}+N u \geq 0 \quad \text { in } Q, \quad p(0) \geq 0, \quad p^{\prime}(0) \geq 0, \\
& u(-p+N u)=0 \\
& p(0) y(0)=0, \quad y^{\prime}(0) p(0)=0, \quad y(0) p^{\prime}(0)=0, \\
& y(0) \int_{0}^{T} A^{\prime}(\sigma) p(\sigma) d \sigma=0 \quad \text { in } \Omega .
\end{aligned}
$$

THEOREM 3.3. Let $J=(1 / 2) \int_{0}^{T}\left|y^{\prime}-z_{d}\right|^{2} d t+\int_{0}^{T}(1 / 2)(N u, u) d t$ and $K=$ $-(1 / 2) \int_{0}^{T}\left|y^{\prime}\right|^{2} d t+(1 / 2) \int_{0}^{T}\left|z_{d}\right|^{2} d t-(1 / 2) \int_{0}^{T}(N u, u) d t$. Assume that $y_{0}, u_{0}$, and $p_{0}$ satisfy (3.20), (3.21), and (3.22), respectively, $y$ and $u$ in $J$ satisfy (3.20), 
and $y$ and $u$ in $K$ satisfy (3.21). Then

$$
\inf _{(3.20)} J=J\left(y_{0}^{\prime}, u_{0}\right)=K\left(y_{0}^{\prime}, u_{0}\right)=\sup _{(3.21)} K
$$

Proof. (i) First, we claim that $J\left(y_{0}^{\prime}, u_{0}\right)=K\left(y_{0}^{\prime}, u_{0}\right)$ :

$$
\begin{aligned}
J\left(y_{0}^{\prime}, u_{0}\right) & =J\left(y_{0}^{\prime}, u_{0}\right)+\int_{0}^{T}\left(p_{0}^{\prime}, u_{0}\right) d t-\int_{0}^{T}\left(N u_{0}, u_{0}\right) d t \\
& =J\left(y_{0}^{\prime}, u_{0}\right)+\int_{0}^{T}\left(p_{0}^{\prime}, y_{0}^{\prime \prime}+A_{2}(t) y_{0}^{\prime}+A_{1}(t) y_{0}\right) d t-\int_{0}^{T}\left(N u_{0}, u_{0}\right) d t \\
& =J\left(y_{0}^{\prime}, u_{0}\right)+\int_{0}^{T}\left(-p_{0}^{\prime \prime}+A_{2}(t) p_{0}^{\prime}-A_{1}(t) p_{0}\right. \\
& \left.=J\left(y_{0}^{\prime}, u_{0}\right)-\int_{0}^{T}\left(y_{0}^{\prime}-z_{d}\right) d t-\int_{0}^{T}(N) p(\sigma) d \sigma, y^{\prime}\right) d t-\int_{0}^{T}\left(N u_{0}, u_{0}\right) d t \\
& =-\frac{1}{2} \int_{0}^{T}\left|y_{0}^{\prime}\right|^{2} d t+\frac{1}{2} \int_{0}^{T}\left|z_{d}\right|^{2} d t-\frac{1}{2} \int_{0}^{T}\left(N u_{0}, u_{0}\right) d t
\end{aligned}
$$

(ii) Second, we must show that $\inf J=J\left(y_{0}^{\prime}, u_{0}\right)$ :

$$
\begin{aligned}
& J\left(y^{\prime}, u\right)-J\left(y_{0}^{\prime}, u_{0}\right) \geq \int_{0}^{T}\left(y_{0}^{\prime}-z_{d}, y^{\prime}-y_{0}^{\prime}\right) d t+\int_{0}^{T}\left(N u_{0}, u-u_{0}\right) d t \\
&= \int_{0}^{T}\left(p_{0}^{\prime \prime}-A_{2}(t) p_{0}^{\prime}+A_{1}(t) p_{0}\right. \\
&\left.\quad+\int_{t}^{T} A_{1}^{\prime}(\sigma) p_{0}(\sigma) d \sigma, y^{\prime}-y_{0}^{\prime}\right) d t \\
& \\
&+\int_{0}^{T}\left(N u_{0}, u-u_{0}\right) d t \\
&=\int_{0}^{T}\left(p_{0}^{\prime},-\left(y^{\prime \prime}-y_{0}^{\prime \prime}\right)-A_{2}(t)\left(y^{\prime}-y_{0}^{\prime}\right)-A_{1}(t)\left(y-y_{0}\right)\right) d t \\
&+\int_{0}^{T}\left(N u_{0}, u-u_{0}\right) d t \\
&=\int_{0}^{T}\left(-p_{0}^{\prime}+N u_{0}, u-u_{0}\right) d t \\
& \geq 0 .
\end{aligned}
$$


(iii) Finally, we check that $\sup K=K\left(y_{0}^{\prime}, u_{0}\right)$ :

$$
\begin{aligned}
& J\left(y_{0}^{\prime}, u_{0}\right)-J\left(y^{\prime}, u\right) \\
& \geq \int_{0}^{T}\left(y^{\prime}-z_{d}, y_{0}^{\prime}, y^{\prime}\right) d t+\int_{0}^{T}\left(N u, u_{0}-u\right) d t \\
&= \int_{0}^{T}\left(p^{\prime \prime}-A_{2}(t) p^{\prime}+A_{1}(t) p+\int_{t}^{T} A_{1}^{\prime}(\sigma) p(\sigma) d \sigma, y_{0}^{\prime}-y^{\prime}\right) d t \\
&-\int_{0}^{T}\left(y_{0}^{\prime \prime}+A_{2}(t) y_{0}^{\prime}+A_{1}(t) y_{0}-u_{0}, p_{0}^{\prime}-p^{\prime}\right) d t+\int_{0}^{T}\left(N u, u_{0}-u\right) d t \\
&=-\int_{0}^{T}\left(y^{\prime}-z_{d}, y^{\prime}\right) d t+\int_{0}^{T}\left(p^{\prime},-y_{0}^{\prime \prime}-A_{2}(t) y_{0}^{\prime}-A_{1}(t) y_{0}\right) d t \\
&-\int_{0}^{T}\left(y_{0}^{\prime},-p_{0}^{\prime \prime}+A_{2}(t) p_{0}^{\prime}-A_{1}(t) p_{0}-\int_{t}^{T} A^{\prime}(\sigma) p_{0}(\sigma) d \sigma\right) d t \\
&+\int_{0}^{T}\left(N u, u_{0}\right) d t-\int_{0}^{T}(N u, u) d t+\int_{0}^{T}\left(u_{0}, p^{\prime}\right) d t \\
&+\int_{0}^{T}\left(u_{0}, p_{0}^{\prime}\right) d t-\int_{0}^{T}\left(u_{0}, p^{\prime}\right) d t \\
& \geq-\int_{0}^{T}\left(y^{\prime}-z_{d}, y^{\prime}\right) d t-\int_{0}^{T}(N u, u) d t+\int_{0}^{T}\left(y_{0}^{\prime}, y_{0}^{\prime}-z_{d}\right) d t \\
&+\int_{0}^{T}\left(y_{0}^{\prime}-z_{d}, y_{0}^{\prime}\right) d t+\int_{0}^{T}\left(N u_{0}, u_{0}\right) d t \\
&+\int_{0}^{T}\left(N u, u_{0}\right) d t+\int_{0}^{T}\left(u_{0}, p_{0}^{\prime}\right) d t \\
&-\int_{0}^{T}\left(u_{0}, p^{\prime}\right) d t+\int_{0}^{T}\left(N u_{0}, u_{0}\right) d t-\int_{0}^{T}\left(N u_{0}, u_{0}\right) d t \\
& T(N u, u) d t
\end{aligned}
$$

This implies that

$$
K\left(y_{0}^{\prime}, u_{0}\right) \geq K(y, u)
$$

So, we claimed Theorem 3.3.

In this case, we observe the terminal value $y^{\prime}(u ; T)$. Since the observation $z(u)$ is given by $y^{\prime}(u ; T)$, the cost functional is given as

$$
J\left(y^{\prime}, u\right)=\frac{1}{2}\left|y^{\prime}(u ; T)-z_{d}\right|^{2}+\frac{1}{2} \int_{0}^{T}(N u, u) d t
$$


We introduce the following systems:

$$
\begin{aligned}
& y^{\prime \prime}(u ; t)+A_{2}(t) y^{\prime}(u ; t)+A_{1}(t) y(u ; t)=u \text { in } Q, \\
& y(u ; 0)=y_{0}, \quad y^{\prime}(u ; 0)=y_{1} \quad \text { in } \Omega, \\
& u \geq 0 \quad \text { a.e. in } Q, \quad y(0) \geq 0, \quad y^{\prime}(0) \geq 0 \quad \text { in } \Omega, \quad y=0 \quad \text { on } \Sigma, \\
& p^{\prime \prime}(u ; t)-A_{2}(t) p^{\prime}(u ; t)+\left(A_{1}(t)-A_{2}^{\prime}(t)\right) p(u ; t)=0 \quad \text { in } Q, \\
& p=0 \quad \text { on } \Sigma, \\
& p(u ; T)=y^{\prime}(T)-z_{d}, \quad p^{\prime}(u ; T)=A_{2}(T) y^{\prime}(T)-z_{d}, \\
& p+N u \geq 0 \quad \text { in } Q, \\
& p(0) \geq 0, \quad p^{\prime}(0) \geq 0 \quad \text { in } \Omega, \\
& u(p+N u)=0, \\
& p(0) y(0)=0, \quad y^{\prime}(0) p(0)=0, \quad y(0) p^{\prime}(0)=0 \quad \text { in } \Omega .
\end{aligned}
$$

THEOREM 3.4. Let $J=(1 / 2)\left|y^{\prime}(T)-z_{d}\right|^{2}+(1 / 2) \int_{0}^{T}(N u, u) d t$ and $K=$ $-(1 / 2)\left|y^{\prime}(T)\right|^{2}+(1 / 2)\left|z_{d}\right|-(1 / 2) \int_{0}^{T}(N u, u) d t$. Assume that $y_{0}, u_{0}$, and $p_{0}$ satisfy (3.29), (3.30), and (3.31), respectively, $y$ and $u$ in $J$ satisfy (3.29), and $y$ and $u$ in $K$ satisfy (3.30). Then

$$
\inf _{(3.29)} J=J\left(y_{0}^{\prime}, u_{0}\right)=K\left(y_{0}^{\prime}, u_{0}\right)=\sup _{(3.30)} K
$$

Proof. (i) First, we show that $J=K$ at $\left(y_{0}^{\prime}, u_{0}, p_{0}\right)$ :

$$
\begin{aligned}
J\left(y_{0}^{\prime}, u_{0}\right)= & J\left(y_{0}^{\prime}, u_{0}\right)-\int_{0}^{T}\left(u_{0}, p_{0}\right) d t-\int_{0}^{T}\left(u_{0}, N u_{0}\right) d t \\
= & J\left(y_{0}^{\prime}, u_{0}\right)-\int_{0}^{T}\left(y_{0}^{\prime \prime}+A_{2}(t) y_{0}^{\prime}+A_{1}(t) y_{0}, p_{0}\right) d t-\int_{0}^{T}\left(N u_{0}, u_{0}\right) d t \\
= & \frac{1}{2}\left|y_{0}^{\prime}(T)-z_{d}\right|^{2}+\frac{1}{2} \int_{0}^{T}\left(N u_{0}, u_{0}\right) d t-\left(y_{0}^{\prime}(T)-z_{d}, y_{0}^{\prime}(T)\right) \\
& -\int_{0}^{T}\left(N u_{0}, u_{0}\right) d t \\
= & -\frac{1}{2}\left|y_{0}^{\prime}(T)\right|^{2}+\frac{1}{2}\left|z_{d}\right|^{2}-\frac{1}{2} \int_{0}^{T}\left(N u_{0}, u_{0}\right) d t \\
= & K\left(y_{0}^{\prime}, u_{0}\right) .
\end{aligned}
$$


(ii) Second, we check that $J\left(y^{\prime}, u\right) \geq J\left(y_{0}^{\prime}, u_{0}\right)$ :

$$
\begin{aligned}
& J\left(y^{\prime}, u\right)-J\left(y_{0}^{\prime}, u_{0}\right) \\
& \geq\left(y_{0}^{\prime}(T)-z_{d}, y^{\prime}(T)-y_{0}^{\prime}(T)\right)+\int_{0}^{T}\left(N u_{0}, u-u_{0}\right) d t \\
&=\left(y_{0}^{\prime}(T)-z_{d}, y^{\prime}(T)-y_{0}^{\prime}(T)\right)+\int_{0}^{T}\left(N u_{0}, u-u_{0}\right) d t \\
&+\int_{0}^{T}\left(p^{\prime \prime}-A_{2}(t) p^{\prime}+\left(A_{1}(t)-A_{2}^{\prime}(t)\right) p, y-y_{0}\right) d t \\
&= \int_{0}^{T}\left(p, y^{\prime \prime}-y_{0}^{\prime \prime}+A_{2}(t) y^{\prime}-A_{2}(t) y_{0}^{\prime}+A_{1}(t) y-A_{1}(t) y_{0}\right) d t \\
&+\int_{0}^{T}\left(N u_{0}, u-u_{0}\right) d t \\
&= \int_{0}^{T}\left(p+N u_{0}, u-u_{0}\right) d t \\
& \geq 0 .
\end{aligned}
$$

(iii) Finally, we claim that $K\left(y^{\prime}, u\right) \leq K\left(y_{0}^{\prime}, u_{0}\right)$ :

$$
\begin{aligned}
& J\left(y_{0}^{\prime}, u_{0}\right)-J\left(y^{\prime}, u\right) \\
& \geq\left(y_{0}^{\prime}(T)-z_{d}, y^{\prime}(T)-y_{0}^{\prime}(T)\right)+\int_{0}^{T}\left(N u_{0}, u-u_{0}\right) d t \\
&=\left(y_{0}^{\prime}(T)-z_{d}, y^{\prime}(T)-y_{0}^{\prime}(T)\right)+\int_{0}^{T}\left(N u_{0}, u-u_{0}\right) d t \\
&+\int_{0}^{T}\left(y_{0}^{\prime \prime}+A_{2}(t) y_{0}^{\prime}+A_{1}(t) y_{0}-u_{0}, p_{0}-p\right) d t \\
&=-\left(y^{\prime}(T)-z_{d}, y^{\prime}(T)\right)-\int_{0}^{T}(N u, u) d t+\left(y_{0}^{\prime}(T)-z_{d}, y^{\prime}(T)\right) \\
&+\int_{0}^{T}\left(N u_{0}, u_{0}\right) d t+\int_{0}^{T}\left(N u+p, u_{0}\right) d t-\int_{0}^{T}\left(p_{0}+N u_{0}, u_{0}\right) d t \\
& \geq-\left(y^{\prime}(T)-z_{d}, y^{\prime}(T)\right)-\int_{0}^{T}(N u, u) d t+\left(y_{0}^{\prime}(T)-z_{d}, y^{\prime}(T)\right) \\
&+\int_{0}^{T}\left(N u_{0}, u_{0}\right) d t .
\end{aligned}
$$

This implies that

$$
K\left(y_{0}^{\prime}, u_{0}\right) \geq K\left(y^{\prime}, u\right)
$$

This completes the proof. 
Acknowledgment. This work was supported by the Korea Research Foundation Grant KRF-2001-037-DA0010.

\section{REFERENCES}

[1] W. L. Chan, Duality in the optimal control of non-well-posed parabolic equations with positive controls, J. Math. Anal. Appl. 107 (1985), no. 2, 509-519.

[2] J.-L. Lions, Optimal Control of Systems Governed by Partial Differential Equations, Die Grundlehren der mathematischen Wissenschaften, vol. 170, SpringerVerlag, New York, 1971.

[3] S.-I Nakagiri and J. Ha, Optimal control problems for hyperbolic distributed parameter systems with damping terms, Ph.D. thesis, Department of Applied Mathematics, Kobe University, Japan, 1996.

[4] J. Y. Park and M. J. Lee, Duality in the optimal control problems for hyperbolic distributed parameter systems with damping terms, J. Math. Anal. Appl. 227 (1998), no. 2, 449-461.

[5]___ Duality in the optimal control of distributed parameter systems governed by hyperbolic equations, Indian J. Pure Appl. Math. 31 (2000), no. 4, 451-460.

[6] _ Duality in the optimal control of hyperbolic equations with positive controls, Int. J. Math. Math. Sci. 23 (2000), no. 3, 181-188.

[7] S. Tanimoto, Duality in the optimal control of non-well-posed distributed systems, J. Math. Anal. Appl. 171 (1992), no. 1, 277-287.

Mi Jin Lee: Department of Mathematics, Pusan National University (PNU), Pusan 609735, Korea

E-mail address: jin8719@hanmai 1 . net

Jong Yeoul Park: Department of Mathematics, Pusan National University (PNU), Pusan 609-735, Korea

E-mail address: jyepark@pusan.ac.kr

Young Chel Kwon: Department of Mathematics, Dong-A University, Pusan 604-714, Korea

E-mail address: yckwun@daunet.donga.ac.kr 


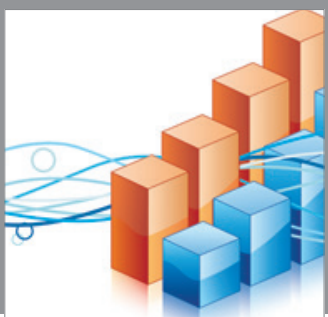

Advances in

Operations Research

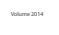

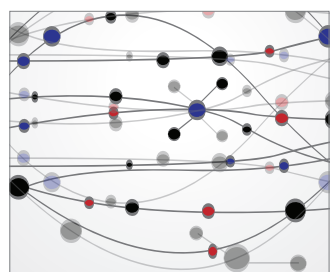

\section{The Scientific} World Journal
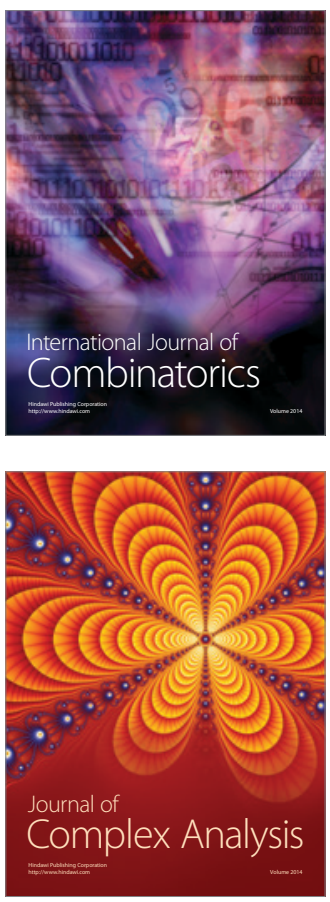

International Journal of

Mathematics and

Mathematical

Sciences
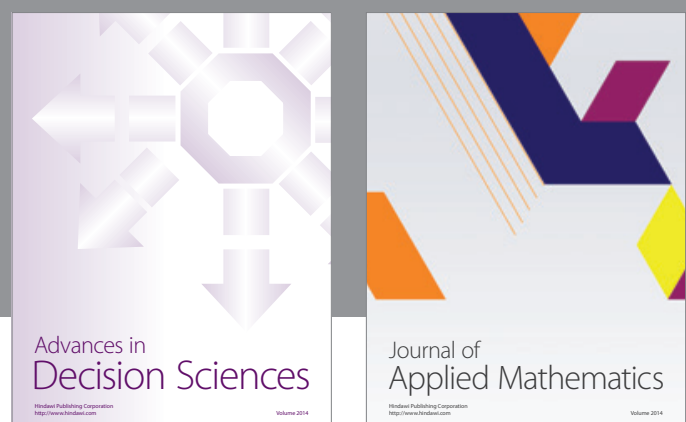

Journal of

Applied Mathematics
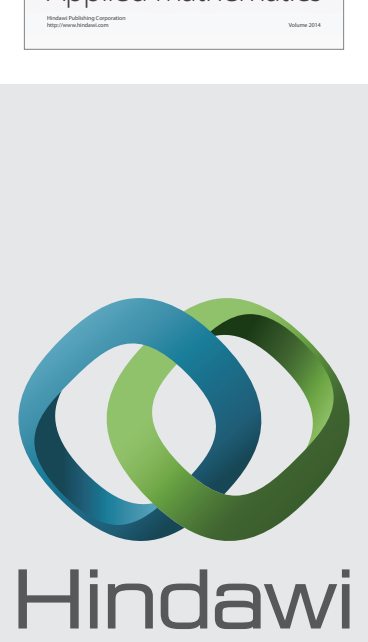

Submit your manuscripts at http://www.hindawi.com
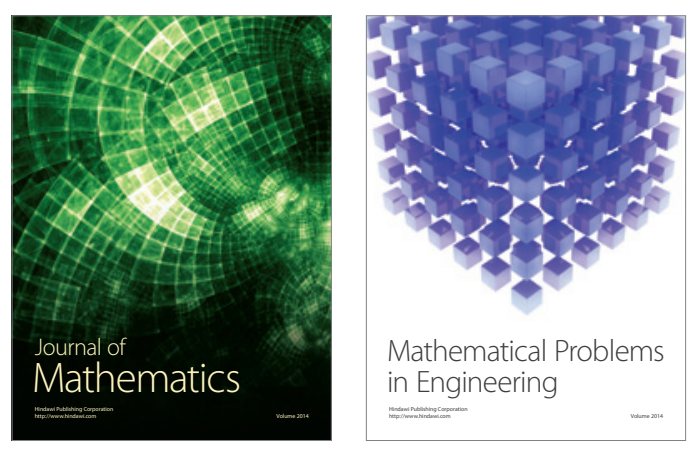

Mathematical Problems in Engineering
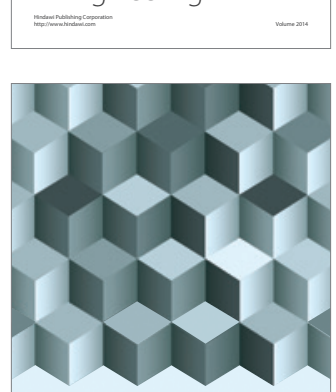

Journal of

Function Spaces
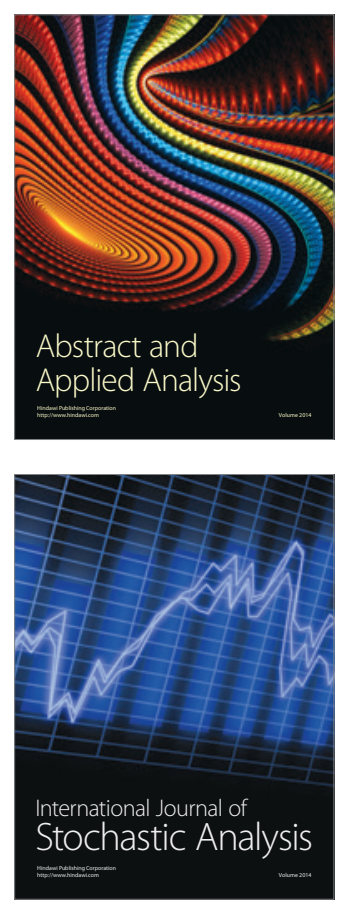

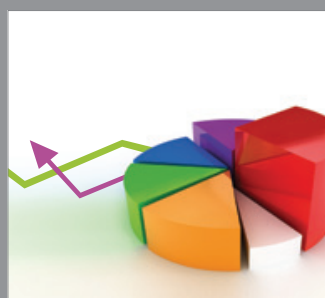

ournal of

Probability and Statistics

Promensencen
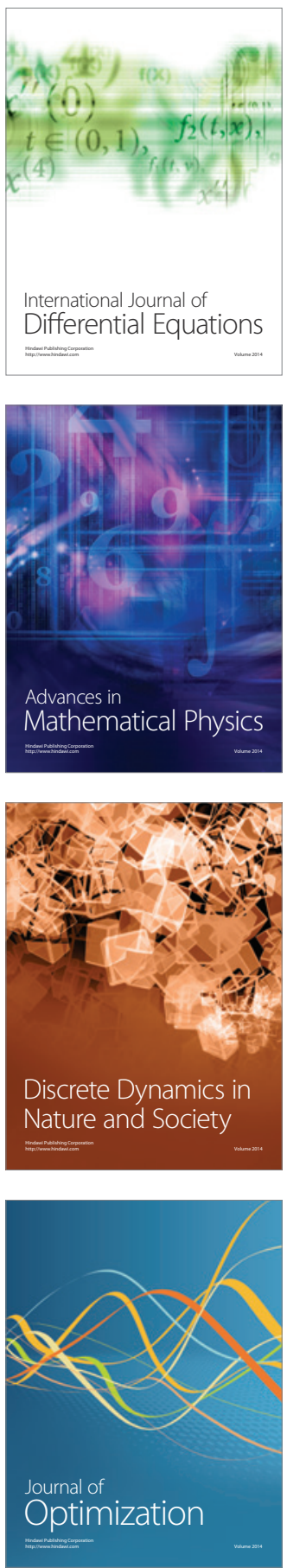